AperTO - Archivio Istituzionale Open Access dell'Università di Torino

Paracrine Cross-Talk Between Human Hair Follicle Dermal Papilla Cells And Microvascular Endothelial Cells.

This is a pre print version of the following article:

Original Citation:

Availability:

This version is available http://hdl.handle.net/2318/1508093

since 2017-05-12T18:26:04Z

Published version:

DOI:10.1111/exd.12670

Terms of use:

Open Access

Anyone can freely access the full text of works made available as "Open Access". Works made available under a Creative Commons license can be used according to the terms and conditions of said license. Use of all other works requires consent of the right holder (author or publisher) if not exempted from copyright protection by the applicable law. 


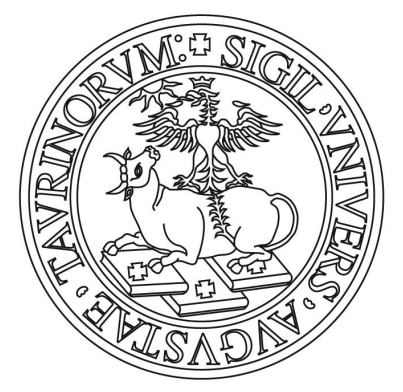

\section{UNIVERSITÀ DEGLI STUDI DI TORINO}

This is an author version of the contribution published on:

Exp Dermatol. 2015 May;24(5):388-90. doi: 10.1111/exd.12670. Epub 2015 Mar 9..

The definitive version is available at:

http://onlinelibrary.wiley.com/doi/10.1111/exd.12670/abstract;jsessionid=D53AA93

B83C7C8B407269CE408C053CD.f01t02 


\title{
PARACRINE CROSS-TALK BETWEEN HUMAN HAIR FOLLICLE DERMAL PAPILLA CELLS AND MICROVASCULAR ENDOTHELIAL CELLS.
}

\author{
E. Bassino ${ }^{1}$, F. Gasparri $^{2}$, V. Giannini ${ }^{2}$ \& L. Munaron ${ }^{1}$
}

${ }^{1}$ Deparment of Life Sciences and System Biology, University of Turin, Italy. ${ }^{2}$ Rottapharm-Madaus, Monza. Italy

\section{Corresponding author:}

Correspondence to:

Luca Munaron, Ph.D.

Dept. Life Sciences \& Systems Biology

University of Torino

Via Accademia Albertina 13

10123 Torino

ITALY

luca.munaron@unito.it 


\section{BACKGROUND}

Human follicle dermal papilla cells (FDPC) are a specialized population of mesenchymal cells located in the skin. They regulate hair follicle (HF) development and growth, and represent a reservoir of multipotent stem cells ${ }^{1}, 2$. Growing evidence supports the hypothesis that HF cycling is associated with vascular remodeling ${ }^{3}$. Follicular keratinocytes release vascular endothelial growth factor (VEGF) that sustains perifollicular angiogenesis leading to an increase of follicle and hair size $^{3}$. Furthermore, several human diseases characterized by hair loss, including Androgenetic Alopecia, exhibit alterations of skin vasculature ${ }^{4,5,6}$. However, the molecular mechanisms underlying HF vascularization remain largely unknown ${ }^{7}$. In vitro co-culture approaches can be successfully employed to greatly improve our knowledge and shed more light on this issue. Here we used Transwell-based co-cultures to show that FDPC promote survival, proliferation and tubulogenesis of human microvascular endothelial cells (HMVEC) more efficiently than fibroblasts. Accordingly, FDPC enhance the endothelial release of VEGF and IGF-1, two well-known proangiogenic growth factors. Collectively, our data suggest a key role of papilla cells in vascular remodeling of the hair follicle ${ }^{8}$.

\section{QUESTIONS ADDRESSED}

The present study provides an experimental validation of quantitative, robust and reliable protocols to investigate paracrine interactions occurring between different cell types associated to hair follicle vascularization by the use of in vitro co-culture approach.

\section{EXPERIMENTAL DESIGN}

See Supplementary material.

\section{RESULTS}

FDPC promote HMVEC survival, proliferation and tubulogenesis and inhibit IL-1 $\alpha$ production.

The maximal HMVEC viability was measured when the cells were grown in DMEM 10\% FCS (see 
Methods and Fig. 1a for setup configurations) and resulted drastically reduced upon serum deprivation (DMEM $0 \%$ or $2 \%$ FCS, 24 h) (Fig. 1b, Supplementary Table 1). In co-culture with FDPC (24/48h), the viability of starved HMVEC was significantly increased (Fig. 1b, Supplementary Table 1). FDPC were also able to enhance HMVEC proliferation upon $48 \mathrm{~h}$ or $72 \mathrm{~h}$ of co-culture: this evidence is confirmed by propidium iodide assay (Fig. 1c, Supplementary Table 1, Supplementary Fig. 1). In addition, FDPC strongly promoted in vitro tubulogenesis of HMVEC cultured in 3D matrigel (Fig. 1d, Supplementary Fig. 2). These functional effects are in nice agreement with the ability of FDPC to release VEGF and IGF-1, two well-known proangiogenic growth factors (Fig. 1e, Supplementary Table 2).

Since IL- $1 \alpha$ is a cytokine prominent in skin wounding and during inflammatory responses, as well as a negative hair growth regulator, we decided to evaluate the effects of FDPC on its production by the endothelium. HMVEC were treated with $\mathrm{H}_{2} \mathrm{O}_{2}(400 \mu \mathrm{M}, 2 \mathrm{~h})$ to induce a strong oxidative stress. In this condition, IL-1 $\alpha$ levels in endothelial medium drastically increased, as expected (24 h) (Fig. 1f). Upon $24 \mathrm{~h}$ of co-culture, FDPC significantly reduced IL-1 $\alpha$ production by HMVEC (Fig. 1f), while no significant change was observed for a prolonged period of co-culture (48 h).

To determine the specificity of the effects exerted by FDPC on HMVEC, we co-cultured endothelium with normal human dermal fibroblasts (NHDF). NHDF actually exhibited a trophic and pro-tubulogenic activity on HMVEC (Supplementary Fig. 3a, b), but at much lower extent when compared to FDPC (Cell viability: $45 \%$ and 19\% of increment from negative control promoted by FDPC and NHDF respectively; Tubulogenesis index: $70 \%$ and $28 \%$ of increment induced by FDPC and NHDF respectively).

Accordingly, VEGF production was less pronounced in fibroblasts compared to FDPC (Supplementary Fig. 3c). Moreover, unlike FDPC, human fibroblasts completely failed to affect IL$1 \alpha$ release triggered by oxidative stress (Supplementary Fig. 3d). 
HMVEC support short-term survival and enhance $\beta$-catenin production by FDPC.

In the complementary configuration (see schemes 1-3 in Fig. 2a), HMVEC sustained FDPC viability only for a short period of co-culture (24h), resulting ineffective at 48h (Fig. 2b). In addition, endothelial cells (EC) were not mitogenic for FDPC (48/72 h; Fig. 2c and Supplementary Table 1).

Finally, we evaluated the production of $\beta$-catenin, a key player in the regulation of follicle cell adhesion and signaling ${ }^{9}$, by quiescent and proliferating FDPC (see detailed Methods in Supplementary material). HMVEC enhanced nuclear $\beta$-catenin expression by FDPC only upon short-term treatment (24 h; Fig. 2d), while no changes were detected for prolonged periods of coculture (48/72h) (Fig. 2e).

\section{CONCLUSIONS}

In this study we employed and validated co-culture technique in order to investigate the paracrine crosstalk between human FDPC and endothelium in vitro. Remarkably, FDPC provide a strong support for endothelial survival, proliferation and tubulogenesis (Fig. 1 and Supplementary Table 1). Accordingly, FDPC release VEGF and IGF-1, two potent proangiogenic mediators whose expression changes during hair growth ${ }^{3},{ }^{10}$ (Supplementary Table 2). Other crucial players in skin development such as human dermal fibroblasts (NHDF) ${ }^{11}$ appear less powerful than FDPC as trophic and protubulogenic sources for endothelium, releasing smaller amount of VEGF. Moreover, NHDF are completely unable to mimic the protective activity of FDPC on HMVEC exposed to oxidative injury, as shown by IL-1 $\alpha$ assays. This observation further highlights the differential paracrine effects of follicle papilla cells and fibroblasts in sustaining skin vasculature (Supplementary Fig. 3).

The relationship between FDPC and HMVEC appears somehow asymmetrical with the inductive power of the first prevailing on the reciprocal one. Indeed, endothelium is not mitogenic and supports only short-time FDPC survival (Fig. 2). 
We acknowledge the limitations of our simplified in vitro setup based on cell culture that takes into account only a part of the complex architecture of native organs. Nonetheless, the validation of this method, that allows to test the effects of pharmacological compounds and drugs by the use of high-throughput and standardized protocols, could successfully integrate and support the well established ex vivo and in vivo approaches ${ }^{12}$.

\section{Acknowledgements}

E.B. performed the research, analysed the data and wrote the paper

F.G. contributed essential reagents or tools and designed the research study

V. G. contributed essential reagents or tools

L.M. designed the research study, analysed the data, wrote the paper and contributed essential reagents or tools.

Conflicts of interest: None declared

\section{REFERENCES}

1 Driskell RR, Clavel C, Rendl M, Watt FM. Hair follicle dermal papilla cells at a glance. $J$ Cell Sci 2011; 124:1179-82.

2 Jahoda C a B, Christiano AM. Niche crosstalk: intercellular signals at the hair follicle. Cell 2011; 146:678-81.

3 Yano K, Brown LF, Detmar M. Control of hair growth and follicle size by VEGF-mediated angiogenesis. J Clin Invest 2001; 107:409-17.

4 Montagna W, Ellis RA. Histology and cytochemistry of human skin. XIII. The blood supply of the hair follicle. J Natl Cancer Inst 1957; 19:451-63.

5 Ellis RA Moretti G. Vascular patterns associated with categen hair follicles in the human scalp. Ann N Y Acad Sci 1959; 83:448-57.

6 Braverman IM Fonferko E. Studies in cutaneous aging: II. The microvasculature. J Invest Dermatol 1982; 78:444-48.

7 Ying Xiao, Wei-Meng Woo, Keisuke Nagao, Wenling Li, Atsushi Terunuma, Yoh-suke, Mukouyama, Anthony E. Oro, Jonathan C. Voge, and Isaac Brownell Perivascular Hair Follicle Stem Cells Associate with a Venule Annulus . J Invest Dermatol 2014; 133:232431.

8 Hill RP, Gardner A, Crawford HC, et al. Human hair follicle dermal sheath and papilla cells support keratinocyte growth in monolayer coculture. Exp Dermatol 2013; 22:236-8.

9 Fagotto F. Looking beyond the Wnt pathway for the deep nature of $\beta$-catenin. EMBO Rep 2013; 14:422-33. 
10 Li W, Man X-Y, Li C-M, et al. VEGF induces proliferation of human hair follicle dermal papilla cells through VEGFR-2-mediated activation of ERK. Exp Cell Res 2012; 318:163340 .

11 Driskell RR, Lichtenberger BM, Hoste E, et al. Distinct fibroblast lineages determine dermal architecture in skin development and repair. Nature 2013; 504:277-81.

12 Jahoda CA, Horne KA OR. Induction of hair growth by implantation of cultured dermal papilla cells. Nature 1984 Oct 11-17; 311:560-2.

\section{FIGURE LEGENDS}

Figure 1. FDPC promote HMVEC survival, proliferation, and tubulogenesis and inhibit IL-1 $\alpha$ production.

A. Scheme of the experimental setup. Arabic numbers in graphs (1-3) are referred to the monocolture and co-culture configurations. 1) HMVEC grown alone, 2) HMVEC grown in presence of the insert filled with cell culture medium only (in the absence of FDPC), 3) HMVEC co-cultured with FDPC.

B. HMVEC viability is maximal in DMEM 10\% FCS and is reduced in DMEM with lower percentage of FCS (0-2\%) (24h). HMVEC survival is significantly increased when co-cultured with FDPC grown in DMEM 2 or $10 \%$ FCS $(24 \mathrm{~h})$. C. HMVEC proliferation is significantly increased when co-cultured with FDPC grown in DMEM 2 or 10\% FCS (48h). Light gray bars (in both survival and proliferative experiments) represent the negative control condition in co-culture: the insert is filled with cell culture medium only (DMEM 0\% FCS or DMEM 10\% FCS) in the absence of FDPC. D. Tubule formation at $18 \mathrm{~h}$ is maximal when HMVEC are grown in Endogro medium and is significantly reduced when grown in DMEM $(0,10 \%)$. Tubulogenesis is significantly increased when HMVEC are co-cultured with FDPC maintained in DMEM 10\% FCS. E. VEGF is maximal when HMVEC are grown in Endogro medium and it is significantly reduced when grown in DMEM (0, 10\% FCS). VEGF levels are significantly increased in the culture medium of HMVEC co-cultured with FDPC for $18 \mathrm{~h}$. F. $\mathrm{H}_{2} \mathrm{O}_{2}(400 \mu \mathrm{M}, 2 \mathrm{~h})$ treatment significantly increases IL-1 $\alpha$ levels in HMVEC (24 h). IL-1 $\alpha$ production is significantly decreased in HMVEC cocultured with FDPC (24h). Only a slight, but not significant, reduction is observed for a prolonged period of co-culture (48h). All values are expressed in A.U. as mean $\pm \mathrm{SEM}$.

Figure 2. HMVEC support short-term FDPC survival and enhance their $\beta$-catenin production.

A. Scheme of the experimental setup. Arabic numbers in graphs (1-3) are referred to the monocolture and co-culture configurations. 1) FDPC grown alone, 2) FDPC grown in presence of the insert filled with cell culture medium only (in the absence of HMVEC), 3) FDPC co-cultured with HMVEC.

B. FDPC viability is maximal in DMEM 10\% FCS and is reduced when grown in DMEM with lower percentage of FCS (0-2\%) (24h). FDPC survival is significantly increased when co-cultured with HMVEC grown in DMEM $10 \%$ FCS $(24 \mathrm{~h})$. C. FDPC proliferation is not modified in coculture with HMVEC grown in DMEM with low percentage of FCS (0-2\%) (48h). In both viability and proliferation assays, light gray bars represent the negative control condition in co-culture: the insert is filled with cell culture medium only (DMEM 0\% FCS or DMEM 10\% FCS) in the absence of HMVEC. Arabic numbers (1-3) are referred to the co-culture configurations. D. HMVEC increase $\beta$-catenin expression by quiescent FDPC only upon short-term treatment (viability culture 
protocol as in figure 1A, $24 \mathrm{~h}$ ), while no significant changes are detected for prolonged periods (48h). E. No significant changes in $\beta$-catenin expression by proliferating FDPC are observed for both $48 \mathrm{~h}$ and $72 \mathrm{~h}$ of co-culture (proliferation culture protocol as in figure $1 \mathrm{C}$ ). All values are expressed in A.U. as mean \pm SEM. 